\title{
Clinical and radiographic evaluations of periodontal intrabony defects treated with enamel matrix derivative: a report of two cases
}

\begin{abstract}
Background: Numerous studies evaluating the clinical and histologic effects of enamel matrix derivative (EMD) as an adjunct to periodontal surgical therapy have demonstrated significant improvements in clinical parameters, radiographic bone fill and periodontal regeneration.
\end{abstract}

Case presentation: Two case reports are described that involved treatment of intrabony defects with periodontal flap surgery and placement of enamel matrix derivative. Each case was followed up after 12 months for clinical and radiographic evaluation. Both cases resulted in a reduction in probing depth (PD), a gain in clinical attachment level $(\mathrm{CAL})$, an absence of bleeding on probing (BOP), unremarkable changes in gingival recession (REC) and radiographic evidence of bone fill.

Conclusion: Treatment of an intrabony defect with periodontal flap surgery and EMD demonstrated bone fill as evidenced by radiographic evaluation performed after 12 months. Consistent improvements in the clinical parameters of CAL, PD and BOP were also noted.

Keywords: Case report, enamel matrix derivative, intrabony defects, radiographic evaluations

\section{Introduction}

Periodontitis is a multifactorial disease. Most of the slight to moderate chronic periodontitis patients respond well to phase I therapy; however, some require surgical intervention. Patients with different types and stages of periodontitis need to be treated with different strategies to ensure the optimal results. Accordingly, a surgical approach may be needed when treating patients with advanced periodontal defects. Surgical therapy may include regenerative treatment which is intended to restore structures destroyed by the disease. ${ }^{1}$ Enamel matrix derivative (EMD) was first introduced in the literature in 1997 and has demonstrated the ability to treat periodontal defects in both humans and animals. ${ }^{2}$ EMD was approved by the U.S. Food and Drug Administration for treatment of periodontal disease by regeneration in angular bony defects. ${ }^{3,4}$ Histologic evidence from human intrabony defects treated with EMD support new attachment on root surfaces denuded by periodontal disease. New cementum and bone formation can occur sub- and supracrestally to a root surface denuded by periodontal disease. ${ }^{5}$ Bhatavadekar et al. treated an intrabony defect by a surgical approach utilizing EMD and demonstrated excellent bone fill as assessed with conventional radiographic examinations in addition to consistent clinical improvements in periodontal parameters including CAL. ${ }^{6}$ Heijl et al. concluded there was no evidence to indicate any clinical adverse effects from the application of EMD in conjunction with periodontal surgery. The procedure will promote an increased gain of radiographic bone and clinical attachment compared to control surgery in the same patient. ${ }^{3}$ No evidence of major differences in clinical outcomes between enamel matrix derivatives and guided tissue regeneration have been reported. EMD appears simpler to use, may not need antibiotic coverage and usually there is no need for a second surgical procedure (as in the case of using a non-resorbable barrier). ${ }^{7}$ Evaluation of the clinical and histologic effects of EMD as an adjunct to non-surgical therapy have demonstrated that periodontal
Volume 10 Issue 2 - 2019

\author{
Zaid R Atarchi,' Mohamed Tarek A Omran,' \\ D Douglas Miley ${ }^{3}$ \\ 'Periodontist working in Private Practice, USA \\ ${ }^{2}$ Department of Graduate Education, Southern Illinois University \\ School of Dental Medicine, USA \\ ${ }^{3}$ Graduate Periodontics, Saint Louis University Center for \\ Advanced Dental Education, Southern Illinois University School
}

of Dental Medicine, USA

Correspondence: Zaid R Atarchi, Periodontist working in Private Practice, Southland Smiles 19815 Governors Hwy

Flossmoor, IL 60422, USA, Tel 773-334-8274,

Email zatarchi@gmail.com

Received: March 12, 2019 | Published: March 18, 2019 regeneration may be attained after the use of enamel matrix derivatives combined with scaling and root planing 8 . In these case reports, two isolated intrabony defects were treated with flap surgery and placement of EMD and evaluated after 12 months by clinical and radiographic examinations

\section{Case I}

\section{Clinical presentation}

A 42- year-old white female with a non-contributory medical history was referred in May 2014 for a periodontal examination and treatment and specifically for a localized increasing pocket depth on maxillary right lateral incisor which had an initial PD of $9 \mathrm{~mm}$ on the disto-palatal aspect and Miller Class I mobility with BOP (Table 1). Bone loss, as determined by the radiograph (Figure 1) was an angular defect. The patient's oral hygiene and plaque control were very good.

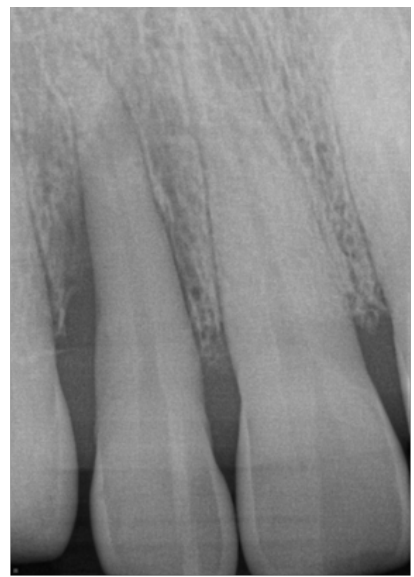

Figure I Pretreatment radiograph of tooth \#7 with angular bony defect on the distal aspect. 
Table I Pocket depth, clinical attachment level and recession measurements $(\mathrm{mm})$ at baseline, 6 months and I 2 months

\begin{tabular}{llllllllll}
\hline & \multicolumn{4}{c}{ Baseline } & \multicolumn{3}{c}{ 6 month } & \multicolumn{3}{c}{ 12 month } \\
\hline Tooth no. & 6 & 7 & 8 & 6 & 7 & 8 & 6 & 7 & 8 \\
Facial PD & 222 & 735 & 524 & 223 & 212 & 323 & 223 & 322 & 212 \\
Palatal PD & 325 & 935 & 545 & 223 & 422 & 334 & 323 & 423 & 323 \\
Facial CAL & 222 & 735 & 524 & 224 & 323 & 423 & 224 & 433 & 312 \\
Palatal CAL & 325 & 935 & 545 & 223 & 422 & 334 & 323 & 423 & 323 \\
Facial REC & 000 & 000 & 000 & 001 & 111 & 100 & 001 & 111 & 100 \\
Palatal REC & 000 & 000 & 000 & 000 & 000 & 000 & 000 & 000 & 000 \\
\hline
\end{tabular}

\section{Case management and clinical outcomes}

Phase I therapy was initiated and consisted of oral hygiene instructions and full mouth scaling and root planing under local anesthesia. Reevaluation was performed after 6 weeks to evaluate the periodontal treatment and the need for any further intervention. Phase II therapy was indicated and included regenerative surgery using enamel matrix derivative to treat the increased probing depth of $9 \mathrm{~mm}$ and residual bony defect on the disto-palatal of tooth \#7. The rationale for treatment was discussed with the patient. She was also informed of the regenerative material and its origin. Because the defect around tooth \#7 was isolated, an endodontic consultation was performed and the likely absence of a root fracture or pulpal pathology was confirmed. No other contributory factors were noted at the examination. The patient was informed of the risk of tissue recession postoperatively as part of the written consent process. The patient was appointed to undergo surgical treatment that was carried out as follows: Local anesthesia utilizing 2\% lidocaine with 1:100,000 epinephrine was administered at the site of the surgery followed by intrasulcular incisions from distal of tooth $\# 5$ to mesial of tooth \#8. A full thickness mucoperioosteal flap was reflected both facially and palatally to give good access to the defect site. The bony defect was identified and all the granulomatous tissue was removed and the root surface was debrided with ultrasonic and hand instruments (Figure $2 \mathrm{a} \& 2 \mathrm{~b}$ ). The intrabony defect was identified as a three-wall distopalatal defect. The root surface was examined for any abnormality or fracture and none was noted. EDTA and enamel matrix derivative were applied to the defect according to the manufacturer instructions. The flap was secured back with interrupted 4-0 chromic gut sutures. Post-operative instructions were given to the patient both written and verbally. The patient was instructed to take pain medication (ibuprofen) as needed and to use chlorhexidine $0.12 \%$ mouth rinse. The patient was seen one week post-operatively and the sutures were removed.

Supportive periodontal therapy was performed for the patient on a regular basis starting 6 weeks after surgery and every three months making sure not to instrument the surgical site subgingivally. The overall plaque control was optimal. The patient was recalled 6 months following surgery. Full-mouth periodontal charting was completed. The disto-palatal surface of tooth \#7 exhibited a remarkable reduction in PD, BOP and mobility (Table 1). The patient was pleased with the esthetics and function at this time. A periapical radiograph obtained and demonstrated bone fill in the treated area with a uniform trabecular bone pattern (Figure 3) 12 months follow-up was performed that revealed consistent clinical findings when they were compared with those at 6 months. The disto-palatal surface of tooth \#7 exhibited a remarkable reduction in PD to $4 \mathrm{~mm}$. There was a CAL gain of $5 \mathrm{~mm}$ (Figure $4 \mathrm{a} \& 4 \mathrm{~b}$ ). Tables 1 present probing measure at baseline, 6 and
12 months and documents a tendency for continued improvement in $\mathrm{PD}$ and CAL. The periapical radiograph at 12 months demonstrates a gain of interproximal bone height in the region between teeth \#6 and \#7 (Figure 5).

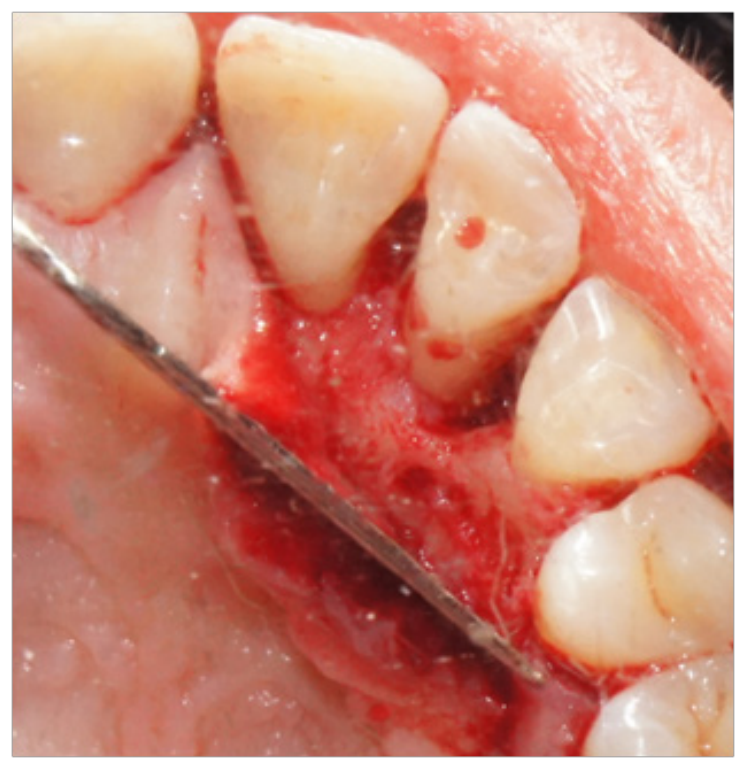

Figure 2a Palatal view of tooth \#7. Reflection of a full-thickness flap showing the bony defect on the distal aspect.

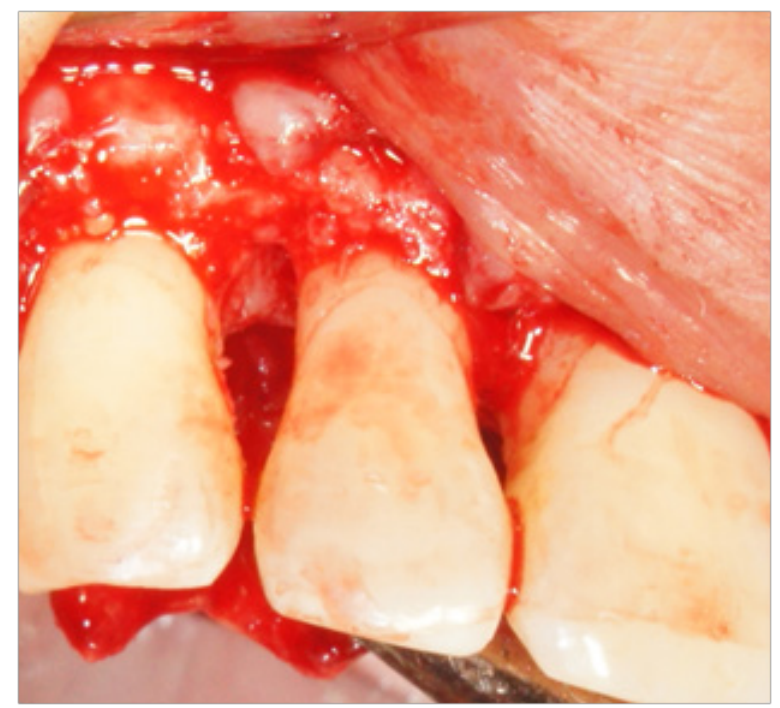

Figure 2b Facial view of tooth \#7. Reflection of a full-thickness flap showing the bony defect on the distal aspect. 


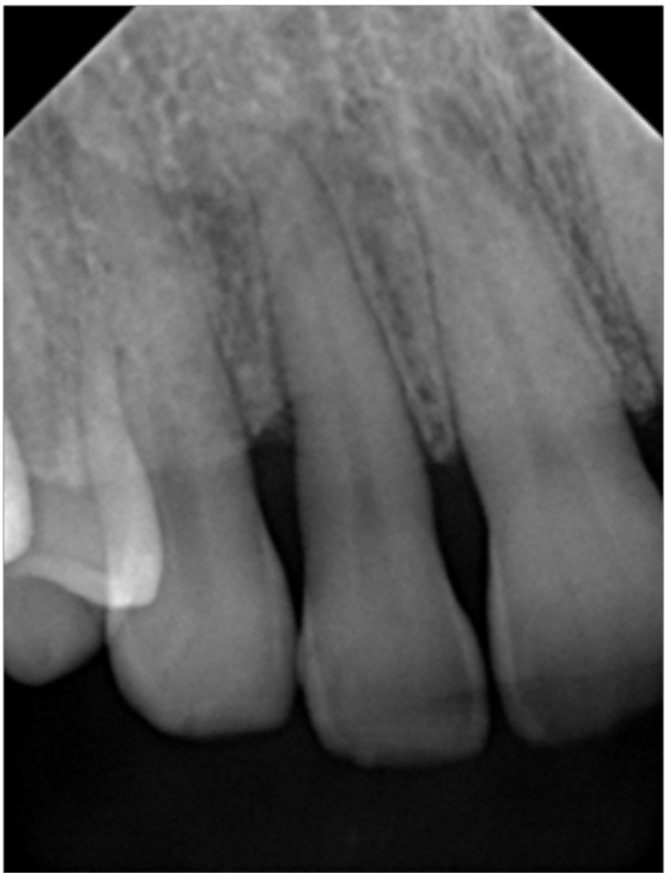

Figure 3 Periapical radiograph of tooth \#7 taken 6 months after surgery demonstrated bone fill in the treated area with a uniform trabecular bone pattern.

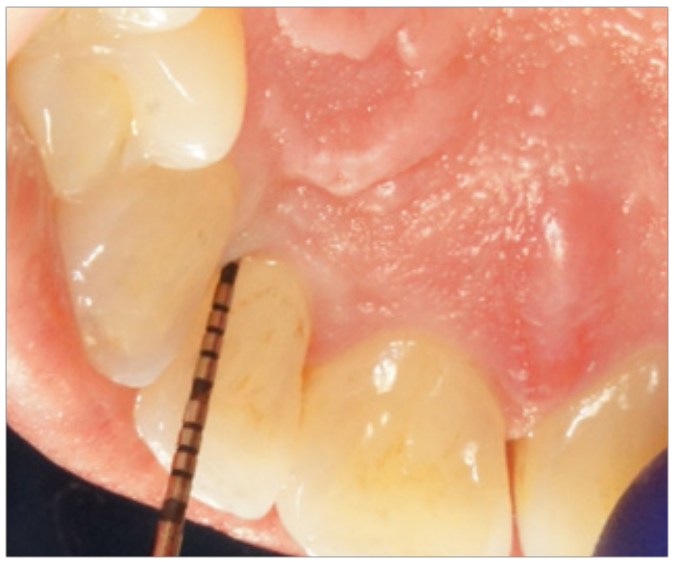

Figure 4a Probing 12 months after surgery reveals PD of $4 \mathrm{~mm}$ on the distopalatal aspect of tooth \#7.

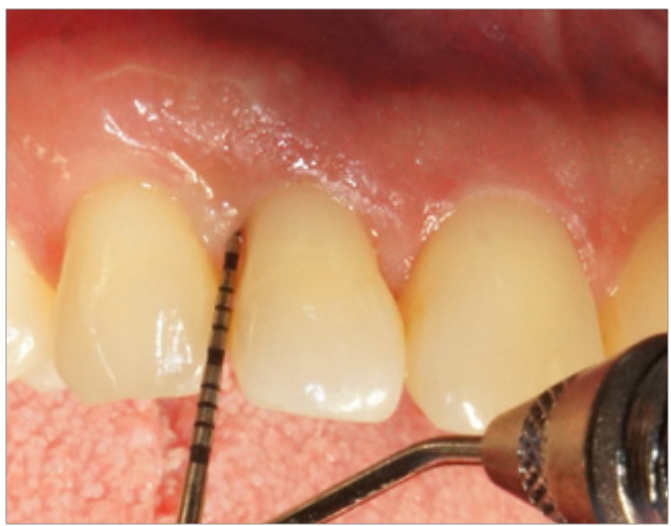

Figure 4b Probing 12 months after surgery reveals PD of $3 \mathrm{~mm}$ on the distofacial aspect of tooth \#7.

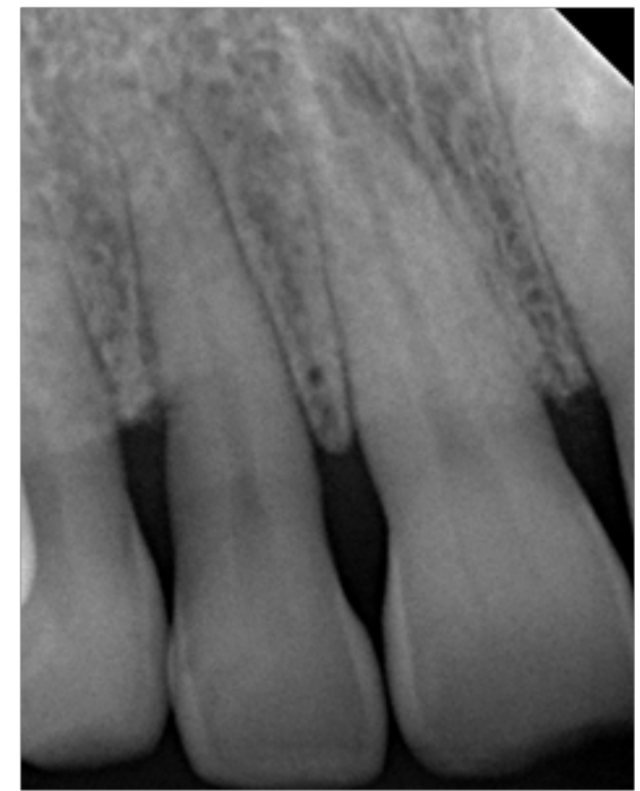

Figure 5 Periapical radiograph of tooth \#7 taken 12 months after surgery demonstrated bone fill in the treated area with a uniform trabecular bone pattern.

\section{Case 2}

\section{Clinical presentation}

A 72 year-old white male was referred due to an isolated increasing probing depth on the disto-facial aspect of the mandibular right lateral incisor which had an initial PD of $10 \mathrm{~mm}$ with BOP (Table 2). Increase in probing depth is illustrated in the clinical photo (Figure 6). The patient was seen for a comprehensive periodontal examination and a periodontal maintenance visit after scaling and root planing was previously performed. His oral hygiene and plaque control was very good and no local factors were detected at that time. Radiographic evaluation revealed a slight radiolucency on the distal of tooth \#26 (Figure 7).

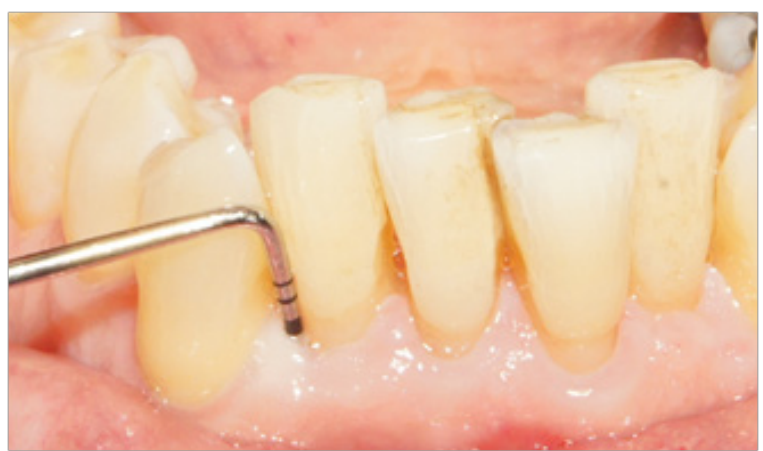

Figure 6 Pretreatment clinical photo of tooth $\# 26$ with distal facial PD of $10 \mathrm{~mm}$.

\section{Management and clinical outcomes}

A decision was made to begin phase II therapy that included regenerative surgery using enamel matrix derivative to treat the increased probing depth of $10 \mathrm{~mm}$ and bony defect on the distofacial of tooth \#26. The rationale for treatment was discussed with the patient and he was informed of the regenerative material and its origin. Because the intrabony defect around tooth \#26 was isolated, 
an endodontic consultation was requested and the likely absence of a root fracture or pulpal pathology was confirmed. No other contributory factors were noted at the baseline examination. The patient was informed of the risk of tissue recession postoperatively as part of the written consent process. The patient was appointed to undergo the surgery that was carried out as follows: Local anesthesia with $2 \%$ lidocaine with 1:100,000 epinephrine was administered at the site of the surgery followed by intrasulcular incisions that were made from mesial of tooth $\# 23$ to mesial of tooth $\# 29$. A full thickness mucoperiosteal flap was reflected both facially and lingually to give good access to the defect site. The bony defect was identified and all the granulomatous tissue was removed and the root surface was debrided with ultrasonic and hand instruments. The bony defect was a two-wall defect (Figure 8). The root surface was examined for any abnormality or fracture and none was noted. EDTA and enamel matrix derivative ${ }^{\text {wa }}$ was applied to the defect according to the manufacturer instructions. The flap was secured back with interrupted 4-0 chromic gut sutures. Post-operative instructions were given to the patient both written and verbally. The patient was instructed to take pain medication (ibuprofen) as needed and to use chlorhexidine $0.12 \%$ as a mouth rinse. The patient was seen 1 week following surgery and the sutures were removed.

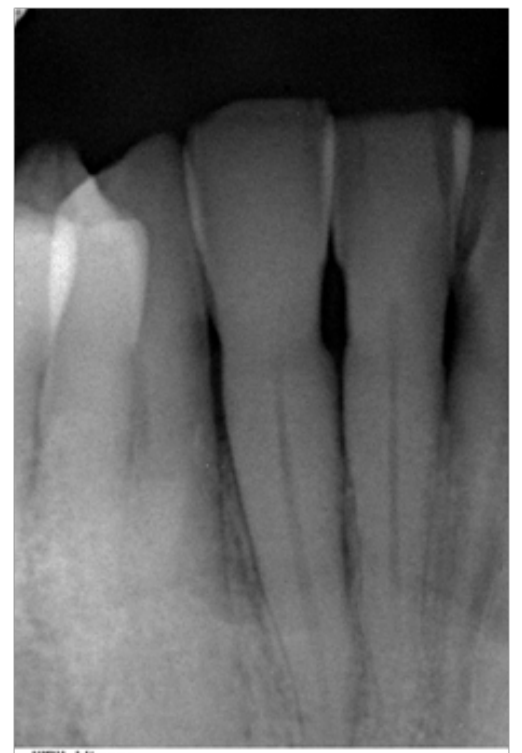

Figure 7 Pretreatment radiograph of tooth \#26 revealed a slight radiolucency on the distal aspect.

Table 2 Pocket depth, clinical attachment level and recession measurements $(\mathrm{mm})$ at baseline, 6 months and I 2 months

\begin{tabular}{llllllllll}
\hline & \multicolumn{4}{c}{ Baseline } & \multicolumn{3}{c}{ 6 month } & \multicolumn{2}{c}{ I2 month } \\
\hline Tooth no. & 27 & 26 & 25 & 27 & 26 & 25 & 27 & 26 & 25 \\
Facial PD & 323 & 1022 & 222 & 323 & 322 & 222 & 322 & 322 & 212 \\
Palatal PD & 212 & 212 & 212 & 212 & 212 & 212 & 222 & 222 & 222 \\
Facial CAL & 323 & 1133 & 232 & 323 & 544 & 333 & 322 & 544 & 323 \\
Palatal CAL & 212 & 323 & 212 & 212 & 323 & 212 & 222 & 333 & 222 \\
Facial REC & 000 & 111 & 010 & 000 & 222 & 111 & 000 & 222 & 111 \\
Palatal REC & 000 & 111 & 000 & 000 & 111 & 000 & 000 & 111 & 000 \\
\hline
\end{tabular}

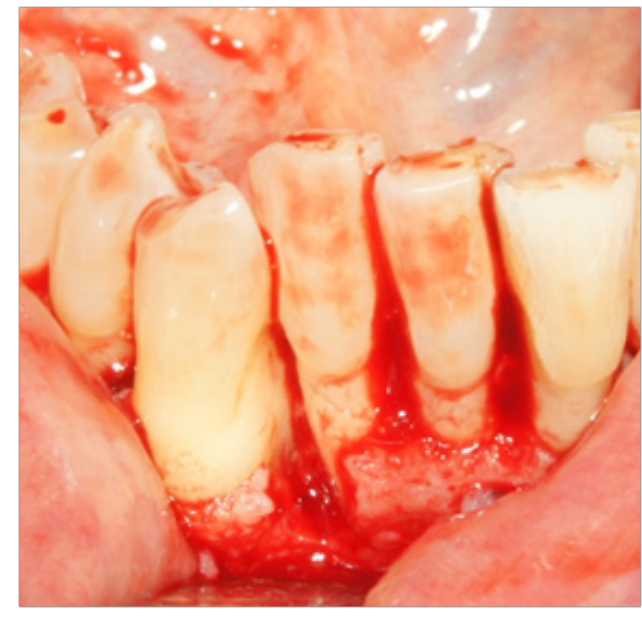

Figure 8 Facial view of tooth \#26. Reflection of a full-thickness flap showing the bony defect on the distal-facial aspect.

Supportive periodontal therapy was performed on a regular basis starting 6 weeks after surgery and every three months making sure not to instrument the surgical site subgingivally. The patient's plaque control was optimal. The patient was recalled 6 months after surgery. Full mouth periodontal charting was completed. The disto-facial surface of tooth \#26 exhibited a remarkable reduction in PD and BOP
(Table 2). The patient was pleased with the esthetics and function. 12 months follow up was performed that revealed stable periodontal readings when they were compared with the 6 months readings. At the 12 month reevaluation, the PD was $3 \mathrm{~mm}$ at the disto-facial surface of \#26. There was a CAL gain of $6 \mathrm{~mm}$ (Figure 9) (Figure 10). Table 2 present clinical measurements at baseline, 6 and 12 months and documents a tendency for continued improvement in PD and CAL from baseline.

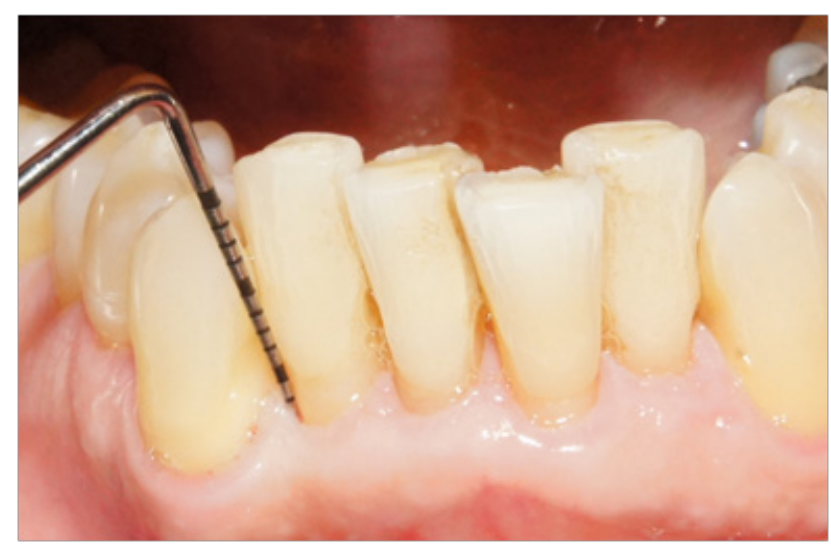

Figure 9 Probing 12 months after surgery reveals PD of $3 \mathrm{~mm}$ on the distofacial aspect of tooth \#26. 


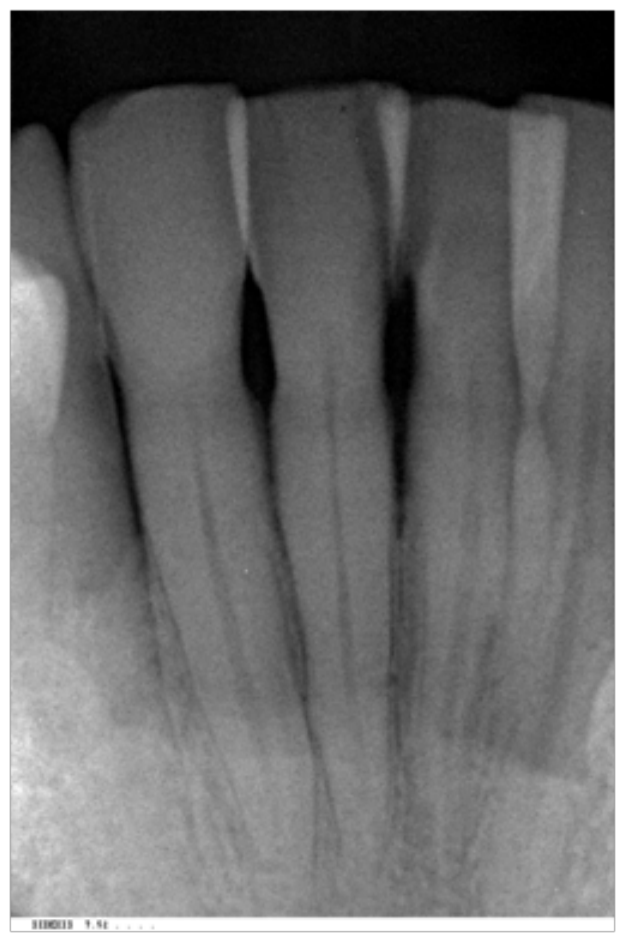

Figure 10 Periapical radiograph of tooth \#26 taken 12 months after surgery.

\section{Discussion}

The findings that treatment of intrabony defects with EMD may result on a short-term basis (up to 1 year) in statistically significantly improvements in PD and CAL gain compared with baseline has been demonstrated by many authors. ${ }^{2,3,8-12}$ The data from previous systematic reviews and clinical studies show that EMD treatment of intrabony defects result in a CAL gain, reduction in PD and a higher radiographic bone density gain compared to conventional open flap debridement procedures. ${ }^{6,11,12}$ Other studies suggest that EMD can be used as an adjunct to scaling and root planing in deep periodontal pockets in which surgery is not feasible or is refused by the patient. ${ }^{8}$ EMD may result in the formation of new cementum, PDL and bone on a previously diseased root surface treated surgically. ${ }^{13}$ EMD procedures are technically more simple and less invasive than GTR, may have fewer complications (membrane exposure and infection), and a limited increase in gingival recession. ${ }^{11}$ Immunogenic potential of EMD application onto the dental root surface in conjunction with periodontal surgery was also tested and revealed very low, if any immunogenic changes. ${ }^{4}$ The cases presented in this report demonstrate favorable results with regard to $\mathrm{CAL}$ gain and $\mathrm{PD}$ improvements using EMD as a biologic material to treat intrabony defects. The initial probing depths at the time of surgery ranged from 2 to $9 \mathrm{~mm}$ for the first case and 2 to $10 \mathrm{~mm}$ for the second case. The configuration of the defect was 3 wall bony defects for the first case and 2-wall bony defect for the second. Although we initially suspected root fracture as an etiology for the isolated defects, this was ruled out intraoperatively. We could not find any notable anatomic or contributory factors besides the presence of some accretion on the root surface. The average CAL gain observed in the first case was $4 \mathrm{~mm}$ as $1 \mathrm{~mm}$ recession was noted after the surgery. The average CAL gain observed in the second case was $6 \mathrm{~mm}$ as $2 \mathrm{~mm}$ recession was noted after the surgery in the defect sites. Previous clinical studies have reported average CAL gains ranging from 2.1 to $4.6 \mathrm{~mm} .^{3,4,9,10}$
There may be some concern that open flap debridement if performed without any regenerative material might have resulted in some amount of bone fill; however, the evidence suggests that the long-term bony defect resolution is greater following the biologic events promoted by the flap access surgery and EMD application. Parodi et al. ${ }^{14}$ evaluated 36-month clinical responses for intrabony defects treated with EMD and documented significant decreases in PD but with net increases in recession. Similarly, Heden and Wennström ${ }^{15}$ observed mean decreases in $\mathrm{PD}$, gains in CAL, but increases in recession at one year following EMD treatment. This case report has limitations. Although we did not use standardized radiographs, we did use standardized exposure parameters and took care to ensure appropriate beam positioning. We also did not use a stent for clinical measurements. Our results only represent two cases, but seem to confirm the results obtained from other clinical trials.

\section{Conclusion}

Treatment of intrabony defects with periodontal flap surgery plus EMD demonstrated bone fill as evidenced by radiographic and clinical examinations performed over 12 months. Consistent improvements in CAL, PD and BOP were also observed with minimal soft tissue recession.

\section{Summary}

\begin{tabular}{ll}
\hline $\begin{array}{l}\text { Why are these } \\
\text { cases new }\end{array}$ & $\begin{array}{l}\text { This successful treatment of intrabony defects was } \\
\text { accomplished with initial non-surgical treatment } \\
\text { information? }\end{array}$ \\
$\begin{array}{l}\text { followed by surgical therapy with the use of enamel } \\
\text { matrix derivative. These cases seem to confirm } \\
\text { previous reports in the scientific literature. }\end{array}$ \\
$\begin{array}{l}\text { What are the } \\
\text { keys to successful } \\
\text { anagement of these } \\
\text { cases? }\end{array}$ & $\begin{array}{l}\text { Adequate flap reflection and root debridement. } \\
\text { Oral hygiene and professional maintenance to } \\
\text { obtain and maintain desirable outcomes. }\end{array}$ \\
$\begin{array}{l}\text { What are the } \\
\text { primary limitations } \\
\text { to success in these } \\
\text { cases? }\end{array}$ & $\begin{array}{l}\text { A compliant and motivated patient who } \\
\text { demonstrates good oral hygiene and will follow } \\
\text { home-care instructions and a professional } \\
\text { maintenance schedule is necessary. }\end{array}$ \\
\hline
\end{tabular}

\$Emdogain, Straumann, Basel, Switzerland

\section{Acknowledgments}

None.

\section{Conflicts of interest}

The author declares that there is no conflict of interest.

\section{References}

1. Greenstein G. Nonsurgical periodontal therapy in 2000: a literature review. J Am Dent Assoc. 2000;131(11):1580-1592.

2. Froum SJ, Weinberg MA, Rosenberg E, et al. A comparative study utilizing open flap debridement with and without enamel matrix derivative in the treatment of periodontal intrabony defects: a 12 -month re-entry study. J Periodontol. 2001;72(1):25-34.

3. Heijl L, Heden G, Svärdström G, et al. Enamel matrix derivative (EMDOGAIN) in the treatment of intrabony periodontal defects. J Clin Periodontol. 1997;24(9 Pt 2):705-714.

4. Zetterström O, Andersson C, Eriksson L, et al. Clinical safety of enamel matrix derivative (EMDOGAIN) in the treatment of periodontal defects. J Clin Periodontol. 1997;24(9 Pt 2):697-704. 
5. Bowers GM, Schallhorn RG, Mellonig JT. Histologic evaluation of new attachment in human intrabony defects. A literature review. $J$ Periodontol. 1982;53(8):509-514.

6. Bhatavadekar NB, Paquette DW. Long-term follow-up and tomographic assessment of an intrabony defect treated with enamel matrix derivative. J Periodontol. 2008;79(9):1802-1808 .

7. Esposito M, Grusovin MG, Papanikolaou N, et al. Enamel matrix derivative (Emdogain $(\mathrm{R})$ ) for periodontal tissue regeneration in intrabony defects. Cochrane Database Syst Rev. 2009;7(4):CD003875.

8. Mellonig JT, Valderrama P, Gregory HJ, et al. Clinical and histologic evaluation of non-surgical periodontal therapy with enamel matrix derivative: a report of four cases. J Periodontol. 2009;80(9):1534-1540.

9. Pontoriero R, Wennström J, Lindhe J. The use of barrier membranes and enamel matrix proteins in the treatment of angular bone defects. A prospective controlled clinical study. J Clin Periodontol. 1999;26(12):833-840.

10. Silvestri M, Ricci G, Rasperini G, et al. Comparison of treatments of infrabony defects with enamel matrix derivative, guided tissue regeneration with a nonresorbable membrane and Widman modified flap. A pilot study. J Clin Periodontol. 2000;27(8):603-610.
11. Zucchelli G, Bernardi F, Montebugnoli L, et al. Enamel matrix proteins and guided tissue regeneration with titanium-reinforced expanded polytetrafluoroethylene membranes in the treatment of infrabony defects: a comparative controlled clinical trial. $J$ Periodontol. 2002;73(1):3-12

12. Cortellini P, Tonetti MS. A minimally invasive surgical technique with an enamel matrix derivative in the regenerative treatment of intrabony defects: a novel approach to limit morbidity. J Clin Periodontol. 2007;34(1):87-93.

13. Windisch P, Sculean A, Klein F, et al. Comparison of clinical, radiographic, and histometric measurements following treatment with guided tissue regeneration or enamel matrix proteins in human periodontal defects. J Periodontol. 2002;73(4):409-417.

14. Parodi R, Santarelli GA, Gasparetto B. Treatment of intrabony pockets with Emdogain: results at 36 months. Int J Periodontics Restorative Dent. 2004;24(1):57-63.

15. Heden G, Wennström JL. Five-year follow-up of regenerative periodontal therapy with enamel matrix derivative at sites with angular bone defects. J Periodontol. 2006;77(2):295-301. 